\title{
RIESGO DE HEPATOTOXICIDAD POR ANTIRRETROVIRALES EN PACIENTES VIH POSITIVO DEL HOSPITAL NACIONAL GUILLERMO ALMENARA IRIGOYEN (HNGAI)
}

\author{
Hepatotoxicity risk for positive HIV patients on antiretroviral therapy in National Hospital \\ Guillermo Almenara Irigoyen
}

Mayte De la Cruz ${ }^{1}$, Marianela Palpa ${ }^{1}$, José R Juárez ${ }^{2}$

${ }^{1}$ Hospital Nacional Guillermo Almenara Irigoyen. ${ }^{2}$ Facultad de Farmácia y Bioquímica. Universidad Nacional Mayor de San Marcos

\section{RESUMEN}

Se revisaron los factores e indicadores de riesgo de hepatotoxicidad en historias clínicas de pacientes VIH positivos que iniciaban un tratamiento antirretroviral de gran actividad (TARGA), entre 1997 a 2003, en el consultorio externo del servicio de Medicina I del Hospital Nacional Guillermo Almenara Irigoyen (HNGAI). La muestra de estudio estuvo conformada por 313 pacientes, de ambos sexos, de 18 a 65 años de edad, con tres a más meses de iniciado el tratamiento antirretroviral. Los factores evaluados fueron: sexo, hepatitis crónica B o C, transaminasas glutámico pirúvica (TGP) y glutámico oxalacética (TGO) basales elevadas e incremento en el conteo de las células CD 4 + después del inicio del TARGA. Los resultados del estudio indican que el $54 \%$ (169 pacientes) de la muestra estudiada desarrollaron hepatotoxicidad, demostrada a través de TGP y TGO elevadas (14,38\% y 15,66\%, respectivamente). En nuestro estudio, mediante la evaluación estadística, no se evidenciaron como factores de riesgo el sexo, hepatitis B o hepatitis C ni el incremento en el conteo de las células $\mathrm{CD}_{4}+$ después del inicio del TARGA. La prevalencia de hepatotoxicidad fue de 53,99\%.

Palabras clave: VIH, hepatotoxicidad, TGO, TGP, VHB, VHC.

\section{SUMMARY}

We reviewed the risk factors and indicators of hepatotoxicity in patient medical records from outpatients HIV $(+)$ who received the first highly active antiretroviral therapy (HAART), from 1997 to 2003, in the doctor's office from the service of Medicine I in the National Hospital Guillermo Almenara Irigoyen (HNGAI). The studied sample was constituted by 313 patients, both genders, 18 to 65 years old, with three and more months after starting antiretroviral therapy. The factors evaluated were: sex, chronic hepatitis B ( $\mathrm{HBV})$ or C (HCV), glutamate pyruvate GPT and glutamate oxaloacetate GOT elevated basal transaminase, increase of CD4+ T-cell count after iniciation of HAART. The results indicate that 54\% (169 patients) from the studied sample (313 patients) developed hepatotoxicity demonstrated through: GPT and GOT basal high (14,38\% y 15,66\%, respectively). For our study, by statistical evaluation, there is not evidence for being a risk factor sex, hepatitis B virus (HBV) and/or hepatitis C virus (HCV) or increase of $\mathrm{CD}_{4}+\mathrm{T}$-cell count at baseline. The prevalence of hepatotoxicity is $53,99 \%$.

Keywords: HIV, hepatotoxicity, GOT, GPT, HBV, HCV.

\section{INTRODUCCIÓN}

A lrededor de 37 millones de adultos están infectados con VIH en todo el mundo y en América Latina 1,6 millones de personas son portadoras. En el Perú, según la Oficina General de Epidemiología del Ministerio de Salud (OGE), hay 17678 casos notificados de SIDA y 23657 personas infectadas por el VIH, a octubre del 2005; siendo reportado, el primer caso de SIDA en el Perú, en $1983^{(1,2)}$. La gran difusión y el acceso a esquemas de tratamiento antirretroviral de gran actividad (TARGA), a partir de la segunda mitad de la década de los 9o, ha propiciado un descenso de $80 \%$ en la mortalidad por VIH después de cuatro años; además permitió abordar problemas relacionados al cumplimiento terapéutico, a la toxicidad, al fracaso inmunológico y virológico del tratamiento y a la aparición de resistencias. Sin embargo, numerosos estudios han identificado la importancia de los efectos adversos asociados al actual tratamiento contra el VIH, según los cuales, al menos el $40 \%$ de los pacientes que empiecen a recibir TARGA sufrirán una o más reacciones adversas; por ejemplo: hepatotoxicidad, exantema, diarrea, anemia o neuropatía periférica, lo que a su vez puede impedir el acceso al tratamiento en entornos con pocos recursos (3-8). Muchos de los fármacos para tratar la infección por VIH se metabolizan en el hígado y pueden causar lesión hepática, entre estos se encuentran los análogos nucleósidos (zidovudina, estavudina y didanosina) y no nucleósidos (efavirenz y nevirapina) e inhibidores de proteasa (Ritonavir) ${ }^{(9-11)}$.

Por tal motivo, el objetivo de nuestro trabajo fue determinar los principales factores e indicadores de riesgo de hepatotoxicidad asociados al tratamiento antirretroviral en pacientes $\mathrm{VIH}(+)$.

\section{MATERIAL Y MÉTODOS}

Se trata de un estudio descriptivo retrospectivo, que se desarrolló en el área del Consultorio Externo de Medicina I del Hospital Nacional Guillermo Almenara Irigoyen (HNGAI). La población de estudio la constituyeron 522 historias clínicas de pacientes $\mathrm{VIH}(+)$ que iniciaban un tratamiento antirretroviral en cualquiera de los esquemas conocidos. De estos, se seleccionaron 313 aplicando los criterios de inclusión: pacientes de ambos sexos, edades entre 18 a 65 años, con un tiempo mayor o igual a tres meses de iniciado el tratamiento 
Tabla 1. Distribución de pacientes según grupos etáreos ( $\bar{x}: 41$ años).

\begin{tabular}{ccc}
\hline $\begin{array}{c}\text { Grupos } \\
\text { etáreos }\end{array}$ & $\mathbf{n}$ & $\mathbf{\%}$ \\
\hline $18-19$ & 2 & 0,64 \\
$20-24$ & 5 & 1,60 \\
$25-29$ & 22 & 7,03 \\
$30-34$ & 57 & 18,21 \\
$35-39$ & 59 & 18,85 \\
$40-44$ & 57 & 18,21 \\
$45-49$ & 39 & 12,46 \\
$50-54$ & 28 & 8,95 \\
$55-59$ & 22 & 7,03 \\
$60-64$ & 7 & 2,24 \\
$>65$ & 15 & 4,78 \\
Total & 313 & 100,00 \\
\hline
\end{tabular}

\begin{tabular}{ccc}
\multicolumn{3}{c}{ Tabla 2. Distribución de pacientes según género. } \\
\hline Género & $\mathbf{n}$ & $\%$ \\
\hline Varones & 218 & 69,65 \\
Mujeres & 95 & 30,35 \\
Total & 313 & 100,00 \\
\hline
\end{tabular}

antirretroviral, con estudio basal de bioquímica hepática y marcadores virales para hepatitis. Se excluyeron a aquellos con enfermedades oportunistas agudas o crónicas, que estaban siendo tratados con otra medicación además de la antirretroviral, o con otras enfermedades hepáticas diferentes a la hepatitis B o C.

Se revisaron las historias clínicas y los resultados de los exámenes clínicos que documentan el seguimiento del paciente en cuanto a bioquímica hepática, marcadores virales para hepatitis y tratamientos antirretrovirales. Se clasificaron los casos de hepatotoxicidad según los grados definidos por AIDS Clinical Trials Group 2, considerando los valores máximos normales de TGO (o-37 U/L) y TGP (o-41 U/L). Los cambios de TGP y TGO serológico desde los niveles de pretratamiento hasta un nivel más alto durante el tratamiento, permitieron categorizar la hepatotoxicidad según una escala estandarizada de cero a cuatro grados. Para el análisis estadístico se utilizó el software SPSS (versión 15,0).

\section{RESULTADOS Y DISCUSIÓN}

En la tabla 1 se muestra la distribución de pacientes por grupos etáreos, siendo la edad media 41 años, con un rango entre 34 a 48, lo cual corrobora lo hallado por Torti y col., que es 38,86 años de edad ${ }^{(12)}$; mientras que los datos de la tabla 2 nos muestran la distribución de pacientes por género, encontrándose que el mayor porcentaje corresponde a los varones, lo cual concuerda con el resumen mundial de la epidemia de VIH/SIDA publicado por ONUSIDA y la OMS ${ }^{(3,13)}$. Este dato sustentaría el porqué, con el transcurso del tiempo, se ha observado un número creciente de víctimas en mujeres heterosexuales que no presentan una vida sexual promiscua y cuyo contagio sería responsabilidad de sus parejas ${ }^{(14)}$.

Los esquemas antirretrovirales principalmente son cinco (Tabla 3), siendo la terapia con el esquema A la de mayor número de pacientes (159), representando el $50,80 \%$ de la muestra de estudio. Este es el esquema con el que generalmente se inicia el tratamiento antirretroviral y que, a través del tiempo, ha mostrado menores reacciones adversas. Al presentarse alguna resistencia este esquema, se siguió con otro más adecuado de acuerdo a cada paciente en particular.

Es importante mencionar que no se encontró significancia estadística entre hepatotoxicidad y esquema de tratamiento, por lo tanto ninguno de los esquemas es un factor de riesgo significativo (Tabla 4); sin embargo debe destacarse que al inicio del tratamiento el $71,38 \%$ de los pacientes tuvieron valores normales de TGO (< $38 \mathrm{U} / \mathrm{L}$ ) frente al $\mathbf{2 8 , 6 2} \%$ que lo tuvieron elevado; con respecto a TGP el 75,52\% tuvieron valores normales $(<41$ $\mathrm{U} / \mathrm{L}$ ) frente al $24,48 \%$ que lo tienen elevado (Tabla 5 ).

La hepatotoxicidad es de inicio brusco y rápido debido, probablemente, a una reacción de hipersensibilidad. El daño hepático, que es progresivo y paulatino, puede deberse a la toxicidad mitocondrial o presentarse por un prolongado tiempo de exposición a los medicamentos antirretrovirales. Patogénicamente, la causa sería el efecto tóxico acumulativo directo inmunomediado ${ }^{(8)}$.

Las enzimas hepáticas elevadas (TGP y TGO) pueden estar relacionadas con toxicidad mitocondrial asociada al uso de inhibidores nucleósidos de la transcriptasa inversa y reacciones de hipersensibilidad asociadas con inhibidores no nucleósidos de la transcriptasa inversa ${ }^{(8,15)}$.

En nuestro estudio encontramos significancia estadística entre hepatotoxicidad, TGO elevada $(\mathrm{p}<0.05)$ y TGP elevada $(\mathrm{p}<0,05)$ (Tablas 6 y 7 ). Para el caso de hepatotoxicidad severa encontramos que el 7,35\% (23) de los pacientes la desarrollaron, siendo el único indicador de riesgo significativo el valor de TGP, no así el de TGO; esto porque si bien la TGO está presente a nivel citosólico y mitocondrial, en el hígado, también lo está en el sistema músculoesquelético, corazón, riñón, cerebro, páncreas, pulmones, leucocitos y glóbulos rojos, siendo menos específica y sensible para el hígado. En cambio la TGP es una enzima citosólica que se encuentra en altas concentraciones (> $41 \mathrm{U} / \mathrm{L})$ en este órgano, por lo cual es más específica de él. La lesión hepatocelular, y no necesariamente la muerte celular, desencadenan la liberación de estas enzimas en la circulación. Ferdinand y colaboradores ${ }^{(16)}$ hallaron como factor de riesgo, 
Tabla 4. Distribución de pacientes según tipo de esquema de tratamiento antirretroviral y presencia de hepatotoxicidad.

\begin{tabular}{|c|c|c|c|c|}
\hline \multirow{3}{*}{$\begin{array}{l}\text { Tipo de } \\
\text { esquema }\end{array}$} & \multicolumn{4}{|c|}{ Hepatoxicidad } \\
\hline & \multicolumn{2}{|c|}{ Presentan } & \multicolumn{2}{|c|}{ No presentan } \\
\hline & $\mathbf{n}$ & $\%$ & $\mathbf{n}$ & $\%$ \\
\hline $\mathrm{A}$ & 91 & 28,44 & 70 & 22,36 \\
\hline B & 17 & 5,75 & 11 & 3,51 \\
\hline $\mathrm{C}$ & 18 & 6,07 & 16 & 5,11 \\
\hline $\mathrm{D}$ & 24 & 7,67 & 27 & 8,63 \\
\hline $\mathrm{E}$ & 8 & 2,56 & 9 & 2,88 \\
\hline $\mathrm{F}$ & 11 & 3,51 & 11 & 3,51 \\
\hline Total & 169 & 54 & 144 & 46 \\
\hline
\end{tabular}

Tabla 5. Distribución de pacientes según niveles de enzimas hepáticas basales.

\begin{tabular}{cccccc}
\hline \multirow{2}{*}{$\begin{array}{c}\text { Enzimas } \\
\text { hepáticas }\end{array}$} & \multicolumn{2}{c}{ Normal } & & \multicolumn{2}{c}{ Elevado } \\
\cline { 2 - 3 } \cline { 5 - 6 } & $\mathbf{n}$ & $\mathbf{\%}$ & & $\mathbf{n}$ & $\mathbf{\%}$ \\
\hline TGO & 223 & 71,38 & & 90 & 28,62 \\
TGP & 236 & 75,52 & & 77 & 24,48 \\
\hline
\end{tabular}

Tabla 6. Distribución de pacientes según TGO basal y presencia de hepatotoxicidad.

\begin{tabular}{|c|c|c|c|c|}
\hline \multirow{3}{*}{ TGO basal } & \multicolumn{4}{|c|}{ Hepatotoxicidad } \\
\hline & \multicolumn{2}{|c|}{ Presentan } & \multicolumn{2}{|c|}{ No presentan } \\
\hline & $\mathbf{n}$ & $\%$ & $\mathbf{n}$ & $\%$ \\
\hline 186 a 370 & 2 & 0,64 & 0 & 0 \\
\hline 93 a 185 & 6 & 1,92 & 0 & 0 \\
\hline 47 a 92 & 41 & 13,10 & 7 & 2,23 \\
\hline 0 a 46 & 120 & 38,34 & 137 & 43,77 \\
\hline Total & 169 & 54 & 144 & 46 \\
\hline
\end{tabular}

Chi cuadrado: 31,41 p<0,05 Existe significación estadística

Tabla 7. Distribución de pacientes según TGP basal y presencia de hepatotixicidad.

\begin{tabular}{cccccc}
\hline \multirow{2}{*}{ TGP basal } & \multicolumn{4}{c}{ Hepatotoxicidad } \\
\cline { 2 - 3 } & \multicolumn{2}{c}{ Presentan } & & \multicolumn{2}{c}{ No presentan } \\
\cline { 2 - 3 } \cline { 5 - 5 } 206 a 410 & 1 & 0,32 & & 0 & n \\
103 a 205 & 9 & 2,88 & & 0 & 0 \\
52 a 102 & 35 & 11,18 & 5 & 1,59 \\
0 a 51 & 124 & 39,62 & & 139 & 44,41 \\
Total & 169 & 54 & & 144 & 46 \\
\hline Chi cuadrado: 31.56 & $\mathrm{p}:<0,05$ & Existe significación estadística
\end{tabular}

para desarrollar grado 4 de hepatotoxicidad, los niveles basales elevados de TGP.

Ningún paciente falleció como resultado directo de hepatoxicidad severa (grados 3 y 4). De 13 pacientes que presentaron el grado cuatro, dos de ellos (15\%) cambiaron de tratamiento antirretroviral, uno por un nuevo fármaco, mientras que en el otro sólo se disminuyó la dosis de Zidovudina; los demás (11) mantuvieron el mismo tratamiento antirretroviral.

Otros datos importantes encontrados en nuestro estudio fueron que el $\mathbf{2 , 2 4} \%$ de los pacientes tienen hepatitis B y $1,60 \%$ hepatitis $C$, valores menores en comparación con los hallados en otros estudios, donde algunos investigadores reportaron 2,7y $52 \%$ para hepatitis
B y $C$, respectivamente, mientras que otros informaron una presencia del $8,8 \%$ para hepatitis B y del $10,7 \%$ para la $C^{(16,17)}$. Esta gran variabilidad tal vez depende de las diferentes poblaciones estudiadas, ya que las alteraciones enzimáticas pueden variar de una región a otra y según el origen étnico de los pacientes.

En lo que se refiere al conteo basal de células $\mathrm{CD}_{4}$, la mediana fue de 232,5 células/ $\mu \mathrm{L}$, no encontrándose significancia estadística entre hepatotoxicidad y $\mathrm{CD}_{4}$ basal.

\section{CONCLUSIONES}

Concluimos que los principales indicadores de riesgo de hepatotoxicidad asociados al tratamiento antirretroviral en pacientes $\mathrm{VIH}$, atendidos en el consultorio externo del servicio de Medicina I del Hospital Nacional Guillermo Almenara Irigoyen (HNGAI), entre los años 1997 y 2003, son: las transaminasas hepáticas basales elevadas. En la muestra estudiada, ningún esquema de tratamiento es un factor de riesgo significativo en el desarrollo de hepatotoxicidad.

\section{REFERENCIAS BIBLIOGRÁFICAS}

1. Alberti G. ONUSIDA: Programa Conjunto de las Naciones Unidas sobre el VIH/SIDA. Informe sobre la epidemia mundial de SIDA, Capítulo 2. ONUSIDA. Ginebra, 2006. p 45-46.

2. Valverde R y Cabello R. Peru In-Country monitoring and evaluation report on the implementation of the UNGASS Declaration of Commitment. A project of the International Council of AIDS Service Organizations (ICASO). Via Libre Association. Diciembre, 2005.

3. UNAIDS. Report on the global AIDS epidemic: 4th global report. UNAIDS/o4.16E, junio de 2004. Programa Conjunto de las Naciones Unidas sobre el VIH/SIDA- ONUSIDA (Versión española). Ginebra, 2004.

4. Project Inform. Estrategias para el manejo de infecciones oportunistas. Maneras de ayudar a prevenir y tratar las infecciones oportunistas. San Francisco, Enero 2007: 6-8. [En línea] Citado en diciembre 2009. Disponible en http:// www.img.thebody.com

5. Dieterich D, Stern J et al. Analyses of four key clinical trials to assess the risk of hepatotoxicity with nevirapine: Correlation with CD4+ levels, Hepatitis B \& C seropositivity, and baseline liver functions tests. The ist IAS Conference on HIV pathogenesis and treatment. Buenos Aires, 2001.

6. Bartlett J. Severe liver toxicity in patients receiving two nucleoside analogues and a non-nucleoside reverse transcriptase inhibitor. 8th Conference on Retroviruses and Opportunistic Infections. Chicago, Febrero 2001.

7. Ministerio de Salud-Comisión Nacional del Sida. Guía Clínica para la atención de las personas adultas que viven con VIH/SIDA. Comunicaciones Mar y Campo Ltda. Santiago de Chile, 2001.

8. Law WP, Dore G, Duncombe CJ et al. Risk of severe hepatotoxicity associated with antiretroviral therapy in the HIV-NAT Cohort, Thailand, 1996-2001. 2003; 17(15): 2191-9.

9. Gutiérrez $\mathrm{R}$ y Soto $\mathrm{A}$. Efectos adversos de la terapia antirretroviral. Instituto de Medicina Tropical Alexander von Humboldt. Universidad Peruana Cayetano Heredia. 
Telemedicina [En línea] Citado en agosto 2009. Disponible en http//:www.upch.edu.pe.tropicales. Lima, 2005.

10. Schiller D. Identification, management and prevention of adverse effects associated with highly active antirretroviral therapy. American Journal of Health-System Pharmacy. December 1, 2004; 61(23):2507-22.

11. Cooper CL. Hepatotoxicity in HIV-HCV coinfection liver histologic damage and antirretroviral drug levels: A Canadian thought-leader perspective. Medscape HIV/AIDS. 2005; 11(1). [En línea] Acceso en agosto 2009. Disponible en: http//:www.medscape.org

12. Torti C, Lapadula G, Casari S et al. Incidence and risk factors for liver enzyme elevation during highly active antiretroviral therapy in HIV-HCV co-infected patients: results from the Italian EPOKA-MASTER Cohort. BMC Infectious Diseases 2005; 5: 58.

13. OPS. Pautas de tratamiento antirretroviral en adultos para países de Latinoamérica y el Caribe: Basadas en las recomendaciones de un grupo consultor y en el documento "Expansión del tratamiento antirretroviral en los entornos con recursos limitados". OPS abril 2002. Washington DC, 2002.

14. Ministerio de Salud. Estado de situación: El SIDA en el Perú al año 200o. Sistema de vigilancia centinela del Programa de Control de Enfermedades de Transmisión Sexual y SIDA
(PROCETSS) del MINSA. Industrial Papirus. Lima, 2001. p. 67.

15. Bonacini M. Liver injury during highly active antirretroviral therapy: The effect of hepatitis $C$ coinfection. Clinical Infectious Diseases 2004; 38 (supl. 2): s104-8.

16. Ferdinand Wit, Weverling GJ, Weel J, Jurriaans S, Lange JM. Incidence of and risk factors for severe hepatoxicity associated with antiretroviral combination therapy. The Journal of Infectious Diseases 2002; 186: 23-31.

17. Sulkowski M, Thomas D, Chaisson R, Moore R. Hepatoxocity associated with antiretroviral therapy in adults infected with Human Immunodeficiency Virus and the role of hepatitis $C$ or B virus infection. JAMA 2000; 283(1): 74-80.

Manuscrito recibido el: 04/02/2011

Aceptado para su publicación el: 05/04/2011

\section{Correspondencia}

Nombre: Dr. José Juárez Eyzaguirre

Dirección: Jr. Los Tulipanes 343 - Lima 12 /

Jr. Puno 1002 - Lima o1 - Perú

e-mail: jjuareze@unmsm.edu.pe 